\title{
Analysis of the Comparative Effectiveness of 3 Oral Bisphosphonates in a Large Managed Care Organization: Adherence, Fracture Rates, and All-Cause Cost
}

\author{
Karen E. Martin, PharmD, MBA, CGP; Jingbo Yu, MHA, PhD; H. Eloise Campbell, BS, PharmD; \\ Jacob Abarca, PharmD, MS; and T. Jeffrey White, PharmD, MS
}

\begin{abstract}
BACKGROUND: Despite widespread availability and use of oral bisphosphonates, fracture rates and associated medical costs are still high. Differences in fracture risk among these agents, if any, have not been quantified due to the lack of high-quality, head-to-head, randomized, controlled trials assessing this outcome. Randomized, placebo-controlled trials have shown that alendronate and risedronate reduce rates of both vertebral and nonvertebral fractures, whereas only reduction in vertebral fractures has been found for ibandronate.
\end{abstract}

OBJECTIVE: To determine if there were any differences among 3 oral bisphosphonates in adherence, total cost of care, and effectiveness in reducing fracture rates in a large managed care population.

METHODS: Administrative, longitudinal pharmacy and medical claims data were obtained from 14 geographically diverse health plans in the United States covering approximately 14 million members. Sampled members had at least 1 pharmacy claim for alendronate, risedronate, or ibandronate during the intake period (January 1, 2005, through 0ctober 31, 2007). The date of the first pharmacy claim for osteoporosis medications within the intake period was the index date. Members were followed for either 12, 24 , or 36 months, depending on length of continuous health plan eligibility. Medication possession ratio (MPR) was measured using a total days supply that was calculated by multiplying the total quantity dispensed by the suggested days supply per unit of dispensing based on manufacturer-recommended dosing. For members who switched bisphosphonate strengths or medications, the estimated days supply was summed for all osteoporosis medications during the follow-up, including overlapping days supply. Outcomes included (a) the first incident fracture and percentages of members with at least 1 fracture after 6 months post-index; (b) the number of days from index to the first incident fracture, measured as time to event in Cox proportional hazards regression analysis; and (c) total all-cause health care costs (health plan allowed amount including member cost share).

RESULTS: A total of 45,939 members were included ( $n=24,909$ alendronate, $n=13,834$ risedronate, $n=7,196$ ibandronate). In the 12-month analysis, MPRs were comparable (means $=0.57-0.58$ ) for the 3 medications. After 24 months, MPRs had dropped for all medications, but those of both alendronate (mean $=0.50,95 \% \mathrm{Cl}=0.49-0.50$ ) and risedronate (mean $=0.50,95 \% \mathrm{Cl}=0.49-0.51$ ) were slightly higher than that of ibandronate (mean $=0.47,95 \% \mathrm{Cl}=0.46-0.48$ ). At 36 months, again the MPRs had dropped for all 3 medications (means $=0.44-0.47$ ) but were similar. There were no statistically significant differences among agents in the percentages of subjects with at least 1 fracture at 12, 24, or 36 months (36-month rates: alendronate $4.41 \%$, risedronate $4.38 \%$, ibandronate $6.28 \%, P=0.102$ ). The numbers of subjects with fracture(s) per month of follow-up were 0.0020 for alendronate, 0.0021 for risedronate, and 0.0022 for ibandronate ( $P=0.087$ overall). However, after adjusting for member characteristics, alendronate users had a $12 \%$ lower risk of experiencing any incident fracture than ibandronate users (hazard ratio $=0.88,95 \%$ $\mathrm{Cl}=0.78-0.99, P=0.034$ ) within the follow-up period. In the first 12 postindex months, ibandronate users had higher mean [SD] unadjusted total all-cause health care costs $(\$ 7,464[\$ 15,975])$ compared with alendronate $(\$ 7,233[\$ 16,671])$ and risedronate $(\$ 6,983[\$ 16,870], P<0.001$ for both comparisons), differences of approximately $\$ 19$ per month and $\$ 40$ per month, respectively. The results of the unadjusted 24-month analysis were similar, but there were no significant cost differences at 36 months. Total cost differences for the 3 medication groups were nonsignificant at 12, 24, and 36 months after adjusting for member characteristics.

CONCLUSIONS: This retrospective analysis of an administrative claims database in a large managed care population showed similar rates of adherence and total adjusted all-cause health care costs for alendronate, risedronate, and ibandronate. Absolute unadjusted rates of fracture were small and did not significantly differ among agents, but after controlling for differences in member characteristics, the risk of fracture was $12 \%$ lower for alendronate users than for ibandronate users.

J Manag Care Pharm. 2011;17(8):596-609

Copyright $\odot 2011$, Academy of Managed Care Pharmacy. All rights reserved.

\section{What is already known about this subject}

- In the United States, osteoporotic fractures accounted for an estimated $\$ 19$ billion in direct costs in 2005, and this figure is expected to rise to $\$ 25.3$ billion by 2025 .

- Randomized, controlled trials (RCTs) of oral bisphosphonates have shown statistically significant reductions in fracture rates versus placebo, but whereas alendronate and risedronate have shown reductions in vertebral and nonvertebral fractures, ibandronate has been shown to reduce vertebral fractures only. Comparison of fracture rates among the bisphosphonates is difficult due to the lack of head-to-head RCTs designed to compare this outcome.

- Several observational studies regarding osteoporosis therapy have been published. A recent analysis by Halpern et al. (2011) showed that low adherence (medication possession ratio [MPR] <80\%) was associated with 37\% higher fracture risks and 12\% higher all-cause medical costs. However, there is a lack of comparative effectiveness data for adherence, fracture rates, and total cost of care among the 3 commonly used oral bisphosphonates (alendronate, risedronate, and ibandronate) for a period longer than 12-18 months. 


\section{Analysis of the Comparative Effectiveness of 3 Oral Bisphosphonates in a Large Managed Care Organization: Adherence, Fracture Rates, and All-Cause Cost}

\section{What this study adds}

- Comparative effectiveness research can help to fill the knowledge gap from RCTs by providing additional evidence about the outcomes of various treatment alternatives, especially comparing newer versus established agents. A large administrative database (pharmacy, medical, and eligibility) was accessed to determine outcomes of treatment with alendronate, ibandronate, and risedronate in a geographically diverse population of 45,939 members who can be considered representative of those eligible to receive oral bisphosphonate therapy in a managed care setting.

- After 24 months, the rates of adherence had dropped for all medications, but those of both alendronate (mean MPR $=0.50$ ) and risedronate (mean MPR $=0.50$ ) were slightly better than that of ibandronate (mean MPR $=0.47$ ). Although unadjusted fracture rates among agents did not significantly differ (numbers of persons with at least 1 fracture $=0.0020-0.0022$ per month of followup), alendronate users had a 12\% lower risk of experiencing any incident fracture than ibandronate users, controlling for other member characteristics.

- Users of ibandronate incurred higher mean all-cause health care costs during the first 12 months of follow-up $(\$ 7,464)$ compared with users of alendronate $(\$ 7,233)$ and risedronate $(\$ 6,983)$, but the adjusted differences became nonsignificant at 12,24 , and 36 months.

- Although the actual adherence and cost differences among the medications were small, the conclusions from this database analysis suggest that alendronate may be associated with a lower adjusted risk of fracture compared with ibandronate.

A bout 10 million Americans are estimated to have osteoporosis. ${ }^{1}$ Although osteoporosis can be secondary to drug use and disease, the most common type is postmenopausal osteoporosis (PMO). Estimates of osteoporotic fracture risk have been placed at 1 in 2 for women and 1 in 4 for men older than age 50 years. In the United States, osteoporotic fractures accounted for an estimated $\$ 19$ billion in direct costs in 2005. Due in part to an aging population, this figure is expected to rise to $\$ 25.3$ billion by $2025 .{ }^{1}$

Several guidelines have been published regarding the prevention, identification, and treatment of osteoporosis. ${ }^{2-4}$ Criteria for treatment decisions are based on assessments of age, gender, bone mineral density (BMD), history of previous fracture, and other risk factors, such as low body mass, smoking status, and oral glucocorticoid use. Oral bisphosphonates are a popular prevention and treatment option. Three oral agents are available in the United States: alendronate, ibandronate, and risedronate. All 3 can be given daily but are more frequently given either once weekly (alendronate and risedronate) or once monthly (ibandronate and risedronate).

Several well-controlled, well-designed, randomized, pla- cebo-controlled studies and a meta-analysis of the oral bisphosphonates have shown significant reduction of fractures in PMO. For alendronate, reductions in hip fracture, vertebral fracture, and wrist fracture were seen within 3 years., ${ }^{5,6}$ Alendronate was also found to be more efficacious in women with PMO after 4 years of treatment when used in higher-risk versus lower-risk subjects. ${ }^{7}$ Risedronate reduced vertebral and nonvertebral (not hip) fractures after 3 years of treatment. ${ }^{8}$ Ibandronate reduced the incidence of new vertebral fractures after 2 years of treatment. ${ }^{9}$ Differences in fracture risk among the agents, if any, have not been quantified due to the lack of head-to-head trials assessing this outcome; however, the agents do have different indications based on the available data from clinical trials. A large head-to-head study is not likely to be performed as it would be very expensive and require the participation of a large number of subjects over an extensive period of time.

Observational studies, including those that are considered to be a form of comparative effectiveness research (CER), can help to fill the evidence gap from randomized controlled trials (RCTs). Several observational studies have recently been published for the bisphosphonates; however, differences in study populations, methodology, and outcomes measured make it difficult to compare the effectiveness of the medications and claims of superiority of one drug over another. ${ }^{10-16}$ For health plans and other decision makers, having a complete picture of how the medication is actually used and the resulting outcomes may prove useful and increase the likelihood of making the most appropriate coverage decisions. Given the lack of headto-head RCTs and the limitations of published observational studies of oral bisphosphonates, a health plan-sponsored retrospective analysis of administrative claims was designed to determine if there were differences among oral bisphosphonate agents in their effectiveness as measured by adherence, fracture rates, or total cost of care in a large managed care population.

\section{Methods}

\section{Data Source}

This study used administrative claims data from the HealthCore Integrated Research Database (HIRD). The HIRD data environment represents a U.S. research network that contains a broad, clinically rich spectrum of longitudinal claims data from 14 health plans in the East, South, Central, and West regions of the United States. It represents claims information from approximately 33 million medical lives, about 1 in 9 Americans. Integrated medical, pharmacy, and eligibility information are linked for approximately 14 million members. Included in this data environment are claims from health maintenance organizations (HMOs), point-of-service (POS) plans, preferred provider organizations (PPOs), and indemnity plans. This study utilized medical and pharmacy claims with dates of service from January 1, 2004, through October 31, 2008. All materials were handled in compliance with the 


\section{Analysis of the Comparative Effectiveness of 3 Oral Bisphosphonates in a Large Managed Care Organization: Adherence, Fracture Rates, and All-Cause Cost}

\section{FIGURE 1 Study Cohort Selection}

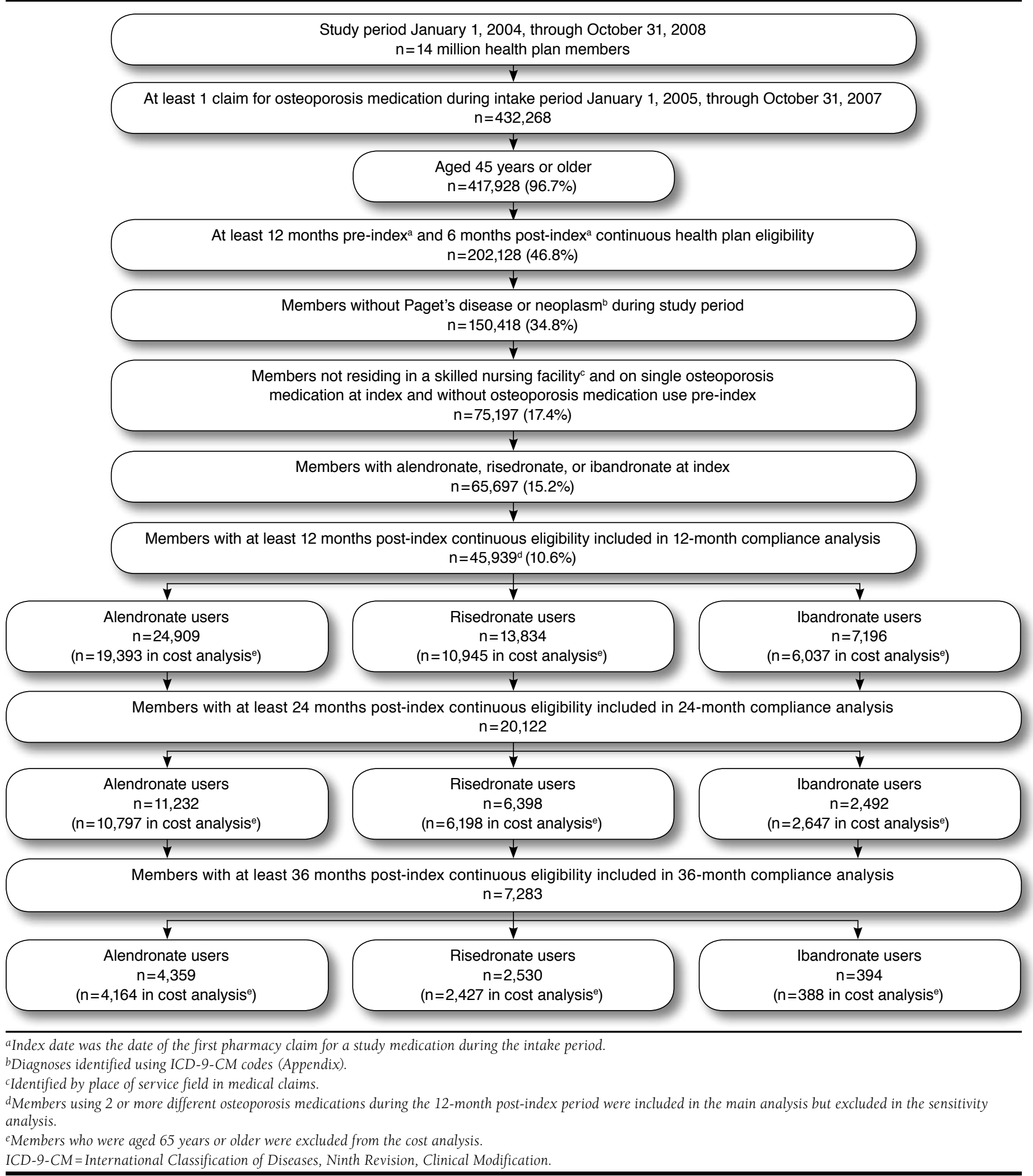




\section{Analysis of the Comparative Effectiveness of 3 Oral Bisphosphonates in a Large Managed Care Organization: Adherence, Fracture Rates, and All-Cause Cost}

Health Insurance Portability and Accountability Act of 1996 (HIPAA). Member confidentially was safeguarded, and data were kept anonymous; therefore, no Institutional Review Board (IRB) approval was sought.

\section{Subject Selection}

Subjects were identified using medical and pharmacy claims data during the intake period from January 1, 2005, through October 31, 2007 (Figure 1). To be included in the study, subjects must have had at least 1 pharmacy claim for at least 1 of the following osteoporosis medications during the intake period: alendronate, risedronate, or ibandronate. Medications were identified by Medi-Span Generic Product Identifier (GPI; Wolters Kluwer Health, Indianapolis, IN) codes (Appendix). Specific dosage form was ignored, and those subjects switching between a daily, monthly, or weekly formulation were counted as continuous users. The date of the first pharmacy claim for osteoporosis medications within the intake period was the index date. Only those subjects with no bisphosphonate medication use in the 12 months pre-index (i.e., newly started on therapy) were included in the sample, except for a sensitivity analysis in which previous bisphosphonate users were included. Subjects were required to (a) have at least 12 months of continuous insurance eligibility preceding the index date and at least 6 months of continuous insurance eligibility after the index date, (b) be aged 45 years or older at index, (c) have no claims for Paget's disease (International Classification of Diseases, Ninth Revision, Clinical Modification [ICD-9-CM] code 731.0) or neoplasm (ICD-9-CM codes 140-208) during the study period, and (d) have no claims with a place of service of skilled nursing facility at index.

Subjects were excluded if they were using 2 or more different osteoporosis medications at index, so that groups would be comparable and control for other osteoporosis medications would not be needed (Figure 1). Members were followed for either 12,24 , or 36 months, depending on length of continuous health plan eligibility. Subjects who used 2 or more different osteoporosis medications during the 12-month post-index period were included in the main analysis but excluded in the corresponding sensitivity analysis. Subjects who were aged 65 years or older were excluded from the cost analysis because reimbursement rates for Medicare and private insurance are different, and Medicare enrollees may use both Medicare and private insurance (possibly with different insurers), thereby providing an incomplete cost picture. In addition, subjects without at least 24 months and 36 months post-index health plan continuous eligibility were excluded from the 24-month and 36-month analyses, respectively.

\section{Outcome Variables}

Medication adherence was measured with medication posses- sion ratio (MPR). Total days supply was calculated for each osteoporosis medication (total quantity dispensed multiplied by the suggested days supply per unit of dispensing based on manufacturer recommended dosing). For example, total days supply for weekly alendronate 35 milligrams (mg) or $70 \mathrm{mg}$ would be calculated by multiplying the quantity dispensed by the recommended 7 days per tablet, and total days supply for daily alendronate $5 \mathrm{mg}$ or $10 \mathrm{mg}$ would be calculated by multiplying the quantity dispensed by the recommended 1 day per tablet. This method has been presented as being more precise than using the days supply field on the pharmacy claim because it does not rely on the submission of the correct days supply by the pharmacy, particularly in studies evaluating dosage forms other than tablets or capsules. ${ }^{17-22}$ The MPR was then calculated by dividing the total days supply of osteoporosis medications by the length of the study interval. For switchers between bisphosphonate strengths or medications, the estimated days supply was summed for all osteoporosis medications during the follow-up, including overlapping days supply. The MPR values were assessed over 12, 24, and 36 months post-index.

The first incident fracture after 6 months post-index was the outcome of interest in risk of fracture analysis. Fracture was defined by skeletal location using validated algorithms designed for use with administrative claims data. ${ }^{23}$ Dorsal and lumbar vertebral fractures were defined using ICD-9-CM diagnosis codes and radiologic procedures (Current Procedural Terminology [CPT]) within 30 days prior to fracture diagnosis. Fractures of the hip, pelvis, femur, lower forearm, radius/ ulna, and humerus were defined using ICD-9-CM diagnosis and a procedure code for repair within 30 days of the fracture diagnosis. Vertebral fracture (not dorsal or lumbar fracture) definition required a procedure code within 30 days prior and a gap of at least 120 days from the last similar fracture claim. All other fractures required an emergency room (ER) visit or gap of at least 120 days from the last similar fracture claim (Appendix). The number of days from index to the first incident fracture was the time-to-event measure in survival analysis using Cox proportional hazards regression analysis.

Total all-cause health care cost (health plan allowed amount) was the major outcome of interest in the cost analysis. Furthermore, costs were reported by service location, including inpatient, ER, office, other outpatient (any outpatient service other than physician visit, e.g., laboratory, radiology, physical therapy, occupational therapy), or skilled nursing facility identified by the place of service field in medical claims. Outpatient pharmacy costs were reported from pharmacy claims. All outcome variables were measured for the time periods of 12 months, 24 months, and 36 months post-index. Costs were adjusted to 2009 U.S. dollars using the Consumer Price Index (CPI) for medical care. 


\section{Analysis of the Comparative Effectiveness of 3 Oral Bisphosphonates in a Large Managed Care Organization: Adherence, Fracture Rates, and All-Cause Cost}

\section{TABLE 1 Baseline Characteristics ${ }^{\mathrm{a}}$ of Subjects by Index Osteoporosis Medication}

\begin{tabular}{|c|c|c|c|c|c|c|c|}
\hline & \multicolumn{2}{|c|}{ Alendronate } & \multicolumn{2}{|c|}{ Risedronate } & \multicolumn{2}{|c|}{ Ibandronate } & \multirow[t]{2}{*}{$P$ Value $^{\mathrm{b}}$} \\
\hline & $\mathrm{n}$ & $\%$ & $\mathrm{n}$ & $\%$ & $\mathrm{n}$ & $\%$ & \\
\hline Subjects & 24,909 & 54.2 & 13,834 & 30.1 & 7,196 & 15.7 & \\
\hline Age (years): mean [SD] & 59.88 & {$[9.46]$} & 59.54 & {$[9.28]$} & 58.60 & {$[8.53]$} & $<0.001$ \\
\hline \multicolumn{8}{|l|}{ Age group (years) } \\
\hline $45-54$ & 7,742 & 31.1 & 4,444 & 32.1 & 2,439 & 33.9 & $<0.001$ \\
\hline $55-64$ & 11,651 & 46.8 & 6,501 & 47.0 & 3,598 & 50.0 & \\
\hline 65 or older & 5,516 & 22.1 & 2,889 & 20.9 & 1,159 & 16.1 & \\
\hline Female & 23,112 & 92.8 & 13,003 & 94.0 & 6,926 & 96.2 & $<0.001$ \\
\hline \multicolumn{8}{|l|}{ Geographic region } \\
\hline East & 6,217 & 25.0 & 3,013 & 21.8 & 1,512 & 21.0 & $<0.001$ \\
\hline South & 5,207 & 20.9 & 3,202 & 23.1 & 1,915 & 26.6 & \\
\hline Central & 4,191 & 16.8 & 2,607 & 18.8 & 1,231 & 17.1 & \\
\hline West & 9,294 & 37.3 & 5,012 & 36.2 & 2,538 & 35.3 & \\
\hline \multicolumn{8}{|l|}{ Health plan type } \\
\hline $\mathrm{HMO}$ & 6,503 & 26.1 & 3,747 & 27.1 & 1,357 & 18.9 & $<0.001$ \\
\hline POS & 1,527 & 6.1 & 813 & 5.9 & 394 & 5.5 & \\
\hline $\mathrm{PPO}$ & 13,221 & 53.1 & 7,524 & 54.4 & 4,768 & 66.3 & \\
\hline FFS & 809 & 3.2 & 437 & 3.2 & 168 & 2.3 & \\
\hline Other & 2,849 & 11.4 & 1,313 & 9.5 & 509 & 7.1 & \\
\hline \multicolumn{8}{|l|}{ Bone mineral density tests } \\
\hline \multicolumn{8}{|l|}{ DXA tests } \\
\hline Any test & 15,039 & 60.4 & 8,452 & 61.1 & 4,624 & 64.3 & $<0.001$ \\
\hline Number of tests: mean [SD] & 0.61 & {$[0.50]$} & 0.62 & {$[0.50]$} & 0.65 & {$[0.50]$} & $<0.001$ \\
\hline \multicolumn{8}{|l|}{ Number of other tests } \\
\hline Any test & 515 & 2.1 & 241 & 1.7 & 158 & 2.2 & 0.035 \\
\hline Number of tests: mean [SD] & 0.02 & {$[0.14]$} & 0.02 & {$[0.13]$} & 0.02 & {$[0.15]$} & 0.037 \\
\hline DCI Score: mean [SD] & 0.41 & {$[0.85]$} & 0.42 & {$[0.87]$} & 0.39 & {$[0.81]$} & $<0.001$ \\
\hline \multicolumn{8}{|l|}{ DCI Score } \\
\hline 0 & 18,444 & 74.0 & 10,242 & 74.0 & 5,307 & 73.7 & $<0.001$ \\
\hline 1 & 4,601 & 18.5 & 2,514 & 18.2 & 1,397 & 19.4 & \\
\hline 2 & 1,069 & 4.3 & 626 & 4.5 & 285 & 4.0 & \\
\hline 3 or more & 795 & 3.2 & 452 & 3.3 & 207 & 2.9 & \\
\hline Pre-index fracture & 2,041 & 8.2 & 1,001 & 7.2 & 584 & 8.1 & 0.003 \\
\hline \multicolumn{8}{|l|}{ Pre-index diagnosisc } \\
\hline Osteoporosis & 13,003 & 52.2 & 7,037 & 50.9 & 3,958 & 55.0 & $<0.001$ \\
\hline Osteopenia & 7,746 & 31.1 & 4,430 & 32.0 & 2,605 & 36.2 & $<0.001$ \\
\hline Arthritis (OA and RA) & 6,610 & 26.5 & 3,691 & 26.7 & 2,095 & 29.1 & $<0.001$ \\
\hline Musculoskeletal pain & 5,913 & 23.7 & 3,358 & 24.3 & 1,917 & 26.6 & $<0.001$ \\
\hline Menopause and menopausal symptoms & 5,811 & 23.3 & 3,359 & 24.3 & 2,059 & 28.6 & $<0.001$ \\
\hline Respiratory diseases & 5,010 & 20.1 & 2,772 & 20.0 & 1,635 & 22.7 & $<0.001$ \\
\hline Hypothyroidism & 4,157 & 16.7 & 2,397 & 17.3 & 1,410 & 19.6 & $<0.001$ \\
\hline Dementia, depression, anxiety, sleep disorders & 2,788 & 11.2 & 1,440 & 10.4 & 924 & 12.8 & $<0.001$ \\
\hline Diabetes & 2,366 & 9.5 & 1,329 & 9.6 & 619 & 8.6 & 0.042 \\
\hline Upper GI disorder & 1,923 & 7.7 & 1,202 & 8.7 & 770 & 10.7 & $<0.001$ \\
\hline Cardiovascular disease & 1,720 & 6.9 & 937 & 6.8 & 480 & 6.7 & 0.749 \\
\hline Cerebrovascular disease & 1,051 & 4.2 & 587 & 4.2 & 300 & 4.2 & 0.968 \\
\hline Indication of decreased mobility & 578 & 2.3 & 319 & 2.3 & 161 & 2.2 & 0.918 \\
\hline Kidney disease & 573 & 2.3 & 313 & 2.3 & 128 & 1.8 & 0.026 \\
\hline Nutritional disorders & 393 & 1.6 & 199 & 1.4 & 153 & 2.1 & 0.001 \\
\hline Hyperthyroidism & 398 & 1.6 & 195 & 1.4 & 133 & 1.8 & 0.051 \\
\hline Obesity & 350 & 1.4 & 158 & 1.1 & 103 & 1.4 & 0.069 \\
\hline Hyperparathyroidism & 155 & 0.6 & 77 & 0.6 & 41 & 0.6 & 0.692 \\
\hline Alzheimer's disease & 74 & 0.3 & 40 & 0.3 & 19 & 0.3 & 0.900 \\
\hline Parkinson's disease & 73 & 0.3 & 40 & 0.3 & 20 & 0.3 & 0.978 \\
\hline Alcohol use disorder & 94 & 0.4 & 30 & 0.2 & 26 & 0.4 & 0.025 \\
\hline Bulimia or anorexia & 36 & 0.1 & 24 & 0.2 & 15 & 0.2 & 0.466 \\
\hline
\end{tabular}




\section{Analysis of the Comparative Effectiveness of 3 Oral Bisphosphonates in a Large Managed Care Organization: Adherence, Fracture Rates, and All-Cause Cost}

\section{TABLE 1 Baseline Characteristics ${ }^{a}$ of Subjects by Index Osteoporosis Medication (continued)}

\begin{tabular}{|c|c|c|c|c|c|c|c|}
\hline & \multicolumn{2}{|c|}{ Alendronate } & \multicolumn{2}{|c|}{ Risedronate } & \multicolumn{2}{|c|}{ Ibandronate } & \multirow[t]{2}{*}{$P$ Value $^{\mathrm{b}}$} \\
\hline & $\mathrm{n}$ & $\%$ & $\mathrm{n}$ & $\%$ & n & $\%$ & \\
\hline \multicolumn{8}{|l|}{ Any use of therapeutic classes below } \\
\hline CV medication & 12,458 & 50.0 & 6,774 & 49.0 & 3,473 & 48.3 & 0.014 \\
\hline Analgesics medication & 9,874 & 39.6 & 5,480 & 39.6 & 3,056 & 42.5 & $<0.001$ \\
\hline Central nervous system agents & 9,257 & 37.2 & 5,212 & 37.7 & 3,225 & 44.8 & $<0.001$ \\
\hline GI medication & 6,110 & 24.5 & 3,632 & 26.3 & 2,174 & 30.2 & $<0.001$ \\
\hline Thyroid hormones & 3,378 & 13.6 & 1,962 & 14.2 & 1,164 & 16.2 & $<0.001$ \\
\hline Estrogen & 3,339 & 13.4 & 2,132 & 15.4 & 1,317 & 18.3 & $<0.001$ \\
\hline Corticosteroids & 3,493 & 14.0 & 2,107 & 15.2 & 1,119 & 15.6 & $<0.001$ \\
\hline Respiratory agents & 2,902 & 11.6 & 1,598 & 11.6 & 942 & 13.1 & 0.002 \\
\hline Antidiabetic medication & 1,591 & 6.4 & 901 & 6.5 & 411 & 5.7 & 0.062 \\
\hline Antineoplastic hormonal agents & 83 & 0.3 & 53 & 0.4 & 20 & 0.3 & 0.446 \\
\hline Smoking cessation medication & 72 & 0.3 & 34 & 0.2 & 37 & 0.5 & 0.003 \\
\hline Number of unique concomitant medications: mean $[S D]^{d}$ & 6.26 & {$[4.66]$} & 6.44 & {$[4.73]$} & 7.03 & {$[5.05]$} & $<0.001$ \\
\hline \multicolumn{8}{|c|}{ 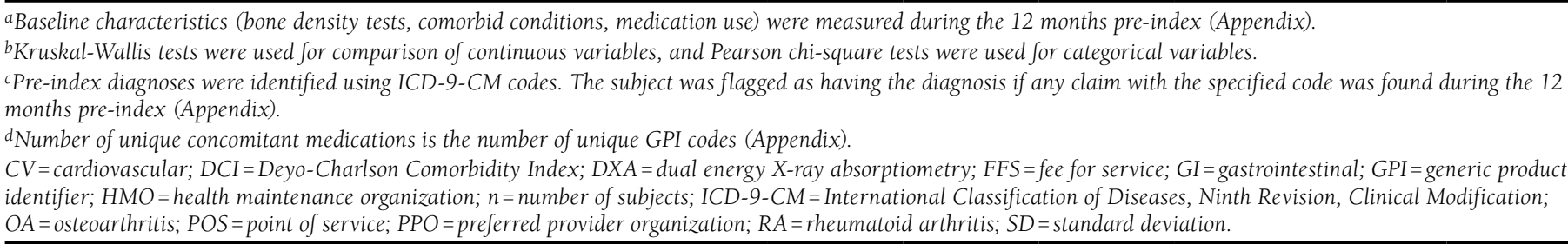 } \\
\hline
\end{tabular}

\section{Other Variables}

Baseline subject demographic characteristics included age at index, gender, region, and type of health plan. Clinical characteristics were measured with Deyo-Charlson Comorbidity Index (DCI) score, number of unique therapeutic classes (unique GPI codes), and a list of related comorbid conditions defined with ICD-9-CM diagnosis codes, the Healthcare Common Procedure Coding System (HCPCS), and/or GPI codes for medication use during the 12 months prior to index, such as fracture, osteoporosis, and arthritis (Appendix). ${ }^{24}$

\section{Statistical Analysis}

Baseline subject characteristics were described and compared among cohorts of alendronate, risedronate, and ibandronate users. The MPR, health care costs, and utilization were reported. Mean and standard deviation (SD) were reported for continuous variables. Frequency and percentage were reported for categorical variables. Kruskal-Wallis tests were used for comparison of continuous variables, and Pearson chi-square tests were used for comparison of categorical variables. Furthermore, generalized linear regression (gamma distribution, log link) was used to evaluate the relationship between bisphosphonate agents and 12-month, 24-month, and 36-month total health care costs, controlling for other subject characteristics. A Cox proportional hazards regression was run to model the relationship between osteoporosis medication use and risk of fracture, adjusting for other patient characteristics. Based on the model, a Kaplan-Meier survival curve was generated to describe the adjusted relationship between osteopo- rosis medication use and risk of fracture over the 36 months post-index. Covariates for the multivariate models included demographics, prior fracture (yes/no), comorbidities, pre-index total health care costs, and other potential confounders. In addition, the following sensitivity analyses were conducted: (a) excluding subjects who switched osteoporosis medications or strengths from the MPR analysis; (b) excluding subjects who switched osteoporosis medications or strengths during the 12 months post-index from the 12-month cost analysis; and (c) including subjects with at least 1 claim for osteoporosis medications during the 12 months pre-index (i.e., "prevalent" osteoporosis subjects) in the cost analysis. All statistical analyses were performed using Stata version 10.1 SE (StataCorp, College Station, TX)

\section{Results}

A total of 45,939 members had at least 1 pharmacy claim for an oral bisphosphonate between January 1, 2005, and October 31, 2007, and met inclusion criteria (Figure 1). The sample included 24,909 alendronate users (54.2\%), 13,834 risedronate users (30.1\%), and 7,196 ibandronate users (15.7\%). The mean ages of the participants in all cohorts were similar, approximately 59-60 years, and more than 92\% were female (Table 1). Statistically significant differences among the groups were noted for age, gender, geographic region, plan type, some comorbidities, and some concomitant medications. Although these absolute differences were small, they were adjusted for in the assessment of fractures and costs. 


\section{Analysis of the Comparative Effectiveness of 3 Oral Bisphosphonates in a Large Managed Care Organization: Adherence, Fracture Rates, and All-Cause Cost}

\section{TABLE 2 Osteoporosis Medication Adherence} by Index Osteoporosis Medication

\begin{tabular}{|c|c|c|c|c|c|}
\hline & $\mathrm{n}$ & Mean & SD & Median & $95 \% \mathrm{CI}$ \\
\hline \multicolumn{6}{|c|}{ During 12-month post-index } \\
\hline \multicolumn{6}{|c|}{ MPR } \\
\hline Alendronate & 24,909 & 0.58 & 0.35 & 0.61 & $0.57-0.58$ \\
\hline Risedronate & 13,834 & 0.58 & 0.36 & 0.61 & $0.57-0.59$ \\
\hline Ibandronate & 7,196 & 0.57 & 0.36 & 0.58 & $0.57-0.58$ \\
\hline \multicolumn{6}{|c|}{ MPR excluding switchers ${ }^{a}$} \\
\hline Alendronate & 16,992 & 0.58 & 0.36 & 0.61 & $0.57-0.58$ \\
\hline Risedronate & 9,299 & 0.58 & 0.36 & 0.61 & $0.57-0.58$ \\
\hline Ibandronate & 4,562 & 0.57 & 0.36 & 0.58 & $0.56-0.58$ \\
\hline \multicolumn{6}{|c|}{ During 24-month post-index } \\
\hline \multicolumn{6}{|c|}{ MPR } \\
\hline Alendronate & 11,232 & 0.50 & 0.36 & 0.46 & $0.49-0.50$ \\
\hline Risedronate & 6,398 & 0.50 & 0.36 & 0.46 & 0.49-0.51 \\
\hline Ibandronate & 2,492 & 0.47 & 0.35 & 0.41 & $0.46-0.48$ \\
\hline \multicolumn{6}{|c|}{ MPR excluding switchers ${ }^{a}$} \\
\hline Alendronate & 8,757 & 0.48 & 0.36 & 0.42 & $0.47-0.49$ \\
\hline Risedronate & 5,332 & 0.50 & 0.37 & 0.46 & $0.49-0.51$ \\
\hline Ibandronate & 2,091 & 0.47 & 0.35 & 0.41 & $0.45-0.48$ \\
\hline \multicolumn{6}{|c|}{ During 36-month post-index } \\
\hline \multicolumn{6}{|c|}{ MPR } \\
\hline Alendronate & 4,359 & 0.44 & 0.35 & 0.36 & $0.43-0.46$ \\
\hline Risedronate & 2,530 & 0.47 & 0.35 & 0.41 & $0.45-0.48$ \\
\hline Ibandronate & 394 & 0.44 & 0.33 & 0.36 & $0.41-0.47$ \\
\hline \multicolumn{6}{|c|}{ MPR excluding switchers ${ }^{a}$} \\
\hline Alendronate & 3,128 & 0.42 & 0.36 & 0.31 & $0.40-0.43$ \\
\hline Risedronate & 1,943 & 0.46 & 0.37 & 0.38 & $0.45-0.48$ \\
\hline Ibandronate & 324 & 0.43 & 0.34 & 0.36 & $0.39-0.47$ \\
\hline \multicolumn{6}{|c|}{$\begin{array}{l}\text { aThe term "switcher" refers to a study subject who switched to a different bisphos- } \\
\text { phonate medication or strength during the post-index follow-up period. } \\
C I=\text { confidence interval; } M P R=\text { medication possession ratio; SD =standard devia- } \\
\text { tion. }\end{array}$} \\
\hline
\end{tabular}

\section{Adherence}

In the 12-month analysis, the rates of adherence were comparable among alendronate (mean $\mathrm{MPR}=0.58$, 95\% confidence interval $[\mathrm{CI}]=0.57-0.58$ ), risedronate (mean $\mathrm{MPR}=0.58$, $95 \% \mathrm{CI}=0.57-0.59$ ), and ibandronate (mean MPR $=0.57,95 \%$ $\mathrm{CI}=0.57-0.58$; Table 2). After 24 months, the mean MPR had dropped for all medications: alendronate $(0.50,95 \% \mathrm{CI}=0.49$ $0.50)$, risedronate $(0.50,95 \% \mathrm{CI}=0.49-0.51)$, and ibandronate (0.47, 95\% CI =0.46-0.48). The MPRs of both alendronate and risedronate were statistically significantly better than that of ibandronate after 24 months, but the clinical significance of this small difference is unclear. By 36 months, again the mean MPR had dropped for all 3 medications: alendronate $(0.44$, $95 \% \mathrm{CI}=0.43-0.46)$, risedronate $(0.47,95 \% \mathrm{CI}=0.45-0.48)$, and ibandronate $(0.44,95 \% \mathrm{CI}=0.41-0.47)$.

The effect of excluding subjects who switched drugs or strengths from the MPR calculation was assessed in a sensitivity analysis (Table 2). There was no difference in MPR between
FIGURE 2 Adjusted Kaplan-Meier Survival Curve by Index Osteoporosis Medications ${ }^{a}$

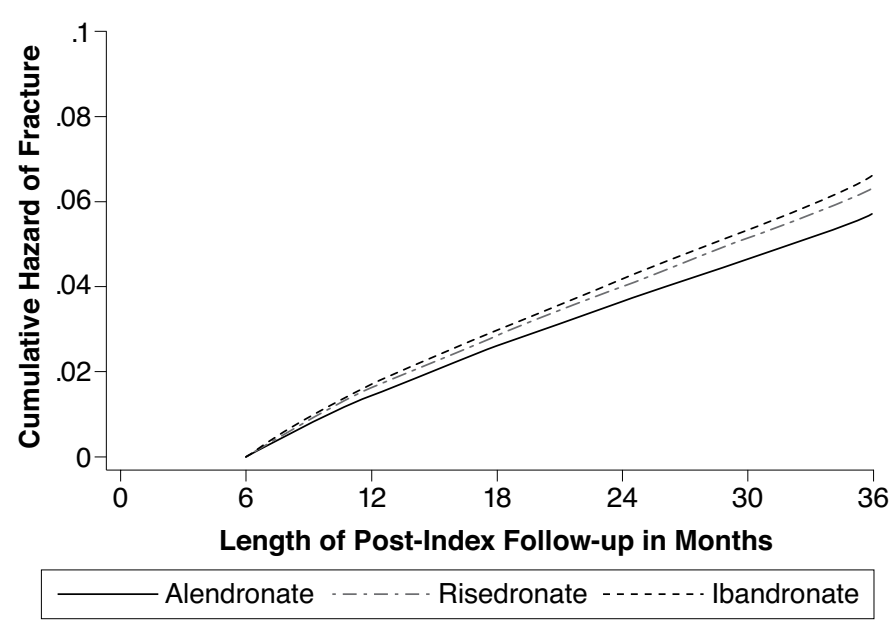

aAdjusted for age, gender, region, plan type, Deyo-Charlson Comorbidity Index score, pre-index comorbidities, and concurrent medication use.

switchers and nonswitchers at 12 months for any medication. At 24 months, only the MPR of alendronate was affected, showing a difference of 0.02 (mean MPR excluding switchers 0.48 , $95 \% \mathrm{CI}=0.47-0.49)$. At 36 months, the MPRs for switchers and nonswitchers differed only slightly for all 3 medications, with differences in mean MPR of 0.01-0.02.

\section{Fracture Risk}

In Cox proportional hazards regression, alendronate users had a $12 \%$ lower risk of experiencing any incident fracture than ibandronate users (hazard ratio $[\mathrm{HR}]=0.88,95 \% \mathrm{CI}=0.78$ $0.99, P=0.034)$, controlling for other subject characteristics (Table 3). There was no significant difference between risedronate and ibandronate $(\mathrm{HR}=0.92,95 \% \mathrm{CI}=0.81-1.04, P=0.183)$. Other factors that were associated with higher fracture risk were older age, female sex, previous fracture, higher DCI score, and polypharmacy. The Kaplan-Meier survival curve describes the relationship between medication use and risk of any fracture following a pharmacy claim for an oral bisphosphonate, adjusting for differences in subject characteristics (Figure 2). The line for ibandronate users was above the line for alendronate users for most of the follow-up time (higher risk), as well.

Absolute differences in rates of fracture were small and did not differ significantly among agents (Table 4). At 12 months, the percentages of subjects with at least 1 fracture were $1.82 \%$ for alendronate, $1.93 \%$ for risedronate, and $2.02 \%$ for ibandronate $(P=0.358)$. There were also no significant differences at 24 months (alendronate $4.30 \%$, risedronate $4.44 \%$, and ibandronate $4.90 \%, P=0.187$ ) or 36 months (alendronate $4.41 \%$, risedronate $4.38 \%$, ibandronate $6.28 \%, P=0.102)$. Measured 


\section{Analysis of the Comparative Effectiveness of 3 Oral Bisphosphonates in a Large Managed Care Organization: Adherence, Fracture Rates, and All-Cause Cost}

TABLE 3 Cox Proportional Hazards Regression for Fracture Risk Analysis from Index Until End of Continuous Plan Eligibility ${ }^{a}$

\begin{tabular}{|c|c|c|c|}
\hline & Hazard Ratio & $95 \%$ CI & $P$ Value \\
\hline \multicolumn{4}{|c|}{ Index osteoporosis medication } \\
\hline Alendronate $(\mathrm{n}=21,802)$ & 0.88 & $0.78-0.99$ & 0.034 \\
\hline Risedronate $(\mathrm{n}=12,062)$ & 0.92 & $0.81-1.04$ & 0.183 \\
\hline Ibandronate $(n=6,316)$ & Reference group & & \\
\hline \multicolumn{4}{|l|}{ Age (years) } \\
\hline $45-54$ & Reference group & & \\
\hline $55-64$ & 1.14 & $1.05-1.23$ & 0.002 \\
\hline 65 or older & 2.15 & $1.96-2.36$ & $<0.001$ \\
\hline \multicolumn{4}{|l|}{ Gender } \\
\hline Female & 1.15 & $1.02-1.29$ & 0.019 \\
\hline Male & Reference group & & \\
\hline \multicolumn{4}{|l|}{ Region } \\
\hline East & Reference group & & \\
\hline South & 0.98 & $0.89-1.08$ & 0.708 \\
\hline Central & 0.89 & $0.80-0.98$ & 0.032 \\
\hline West & 0.91 & $0.83-0.99$ & 0.038 \\
\hline
\end{tabular}

Plan type

\begin{tabular}{l|c|c|c}
\hline HMO & Reference group & & \\
\hline POS & 1.16 & $1.00-1.35$ & 0.044 \\
\hline PPO & 1.32 & $1.21-1.43$ & $<0.001$ \\
\hline FFS & 2.06 & $1.83-2.32$ & $<0.001$ \\
\hline Other & 1.36 & $1.20-1.53$ & $<0.001$ \\
\hline $\begin{array}{l}\text { Pre-index any bone } \\
\text { density test }\end{array}$ & 0.94 & $0.88-1.00$ & 0.050 \\
\hline Pre-index any fracture & 2.65 & $2.47-2.85$ & $<0.001$ \\
\hline
\end{tabular}

Pre-index DCI score

\begin{tabular}{l|c|c|c}
\hline 0 & Reference group & & \\
\hline 1 & 1.27 & $1.19-1.36$ & $<0.001$ \\
\hline 2 & 1.49 & $1.34-1.66$ & $<0.001$ \\
\hline 3 and above & 1.60 & $1.42-1.81$ & $<0.001$ \\
\hline $\begin{array}{l}\text { Pre-index osteopenia } \\
\text { diagnosis }\end{array}$ & 0.91 & $0.84-0.98$ & 0.009 \\
\hline $\begin{array}{l}\text { Pre-index arthritis (OA and } \\
\text { RA) diagnosis }\end{array}$ & 1.15 & $1.08-1.22$ & $<0.001$ \\
\hline $\begin{array}{l}\text { Pre-index musculoskeletal } \\
\text { pain diagnosis }\end{array}$ & 1.27 & $1.19-1.35$ & $<0.001$ \\
\hline $\begin{array}{l}\text { Pre-index menopause and } \\
\text { menopausal symptoms }\end{array}$ & 0.84 & $0.78-0.91$ & $<0.001$ \\
\hline $\begin{array}{l}\text { Pre-index dementia, } \\
\text { depression, anxiety, sleep } \\
\text { disorders }\end{array}$ & 1.33 & $1.23-1.44$ & $<0.001$ \\
\hline $\begin{array}{l}\text { Pre-index GI disorder } \\
\begin{array}{l}\text { Pre-index cardiovascular } \\
\text { disease }\end{array}\end{array}$ & 1.14 & $1.04-1.24$ & 0.004 \\
$\begin{array}{l}\text { Pre-index number of } \\
\text { therapeutic classes 5 or more } \\
\text { (reference group: 0-4) }\end{array}$ & 1.17 & $1.10-1.25$ & $<0.001$ \\
\hline $\begin{array}{l}\text { Pre-index total costs } \\
\text { \$1,000, 2009 U.S. dollars) }\end{array}$ & 1.00 & $1.00-1.00$ & $<0.001$ \\
\hline
\end{tabular}

${ }^{a}$ Model goodness of fit was tested with Score test (chi2[9] $\left.=20.489, P=0.015\right)$ and Likelihood Ratio Test (LR chi2[9] $=20.626, P=0.014)$.

$C I=$ confidence interval; $D C I=$ Deyo-Charlson Comorbidity Index; FFS $=$ fee for service; $G I=$ gastrointestinal; $H M O=$ health maintenance organization; $O A=0$ steoarthritis; $P O S=$ point of service; $P P O=$ preferred provider organization; $R A=r$ heumatoid arthritis. as numbers of persons with fracture(s) per month of followup, rates of fracture were 0.0020 for alendronate, 0.0021 for risedronate, and 0.0022 for ibandronate $(P=0.087$ overall; Table 5).

\section{Costs}

The total health care costs (including those unrelated to osteoporosis) incurred to manage a member on an oral bisphosphonate are displayed in Table 4. During the 12 months post-index, users of ibandronate incurred significantly higher mean [SD] costs $(\$ 7,464[\$ 15,975])$ compared with both alendronate $(\$ 7,233[\$ 16,671])$ and risedronate $(\$ 6,983[\$ 16,870]$, $P<0.001)$ users. Medical costs represented more than one-half of the total costs in all 3 cohorts (67.5\% for alendronate, $66.1 \%$ for risedronate, and $63.9 \%$ for ibandronate). For the costs by service location, significantly higher costs were seen among ibandronate users for office place of service (means of $\$ 778$, $\$ 674$, and $\$ 676$ for ibandronate, alendronate, and risedronate, respectively, $P<0.001$ ), other outpatient (means of $\$ 2,476$, $\$ 2,408$, and $\$ 2,210$, respectively, $P<0.001$ ), and pharmacy (means of $\$ 2,697, \$ 2,351$, and $\$ 2,368$, respectively, $P<0.001$ ). Ibandronate users incurred significantly lower skilled nursing facility costs (means of $\$ 9, \$ 29$, and $\$ 16$, respectively, $P<0.001$ ). Overall, the biggest cost drivers in all 3 cohorts were other outpatient and pharmacy charges. Costs for services billed with an inpatient place of service comprised the third largest outlay.

In generalized linear regression, the difference in total costs at 12 months among the 3 medication groups trended in the same direction, but these were not significant when adjusting for subject characteristics (Table 6). Older age, male gender, East region, higher DCI score, pre-index diagnosis of arthritis or musculoskeletal pain, and higher pre-index total costs were associated with higher 12-month post-index total costs. The sensitivity analyses for prevalent osteoporosis subjects and subjects without osteoporosis medication switching at 12 months produced similar results without significant total cost differences among medications (data not shown).

Ibandronate users had significantly higher total mean [SD] unadjusted health care costs than alendronate users during 24 months post-index $(\$ 14,873[\$ 26,917]$ vs. $\$ 13,787$ [ $\$ 28,298]$, respectively, $P<0.001$ ), but the differences were not significant at 36 months $(\$ 23,190$ [\$41,083] vs. $\$ 21,338$ [\$46,665], $P=0.478$; Table 4). After adjustment for subject characteristics using generalized linear regression, between-group differences in total health care costs were insignificant at both 24 and 36 months (Table 6).

\section{Discussion}

This study is among one of the largest observational database analyses to describe adherence, fracture risk, and total health care utilization and costs in a managed care population using 


\section{Analysis of the Comparative Effectiveness of 3 Oral Bisphosphonates in a Large Managed Care Organization: Adherence, Fracture Rates, and All-Cause Cost}

\section{TABLE 4 Health Care Utilization, Costs, and Fracture Rates by Index Osteoporosis Medications}

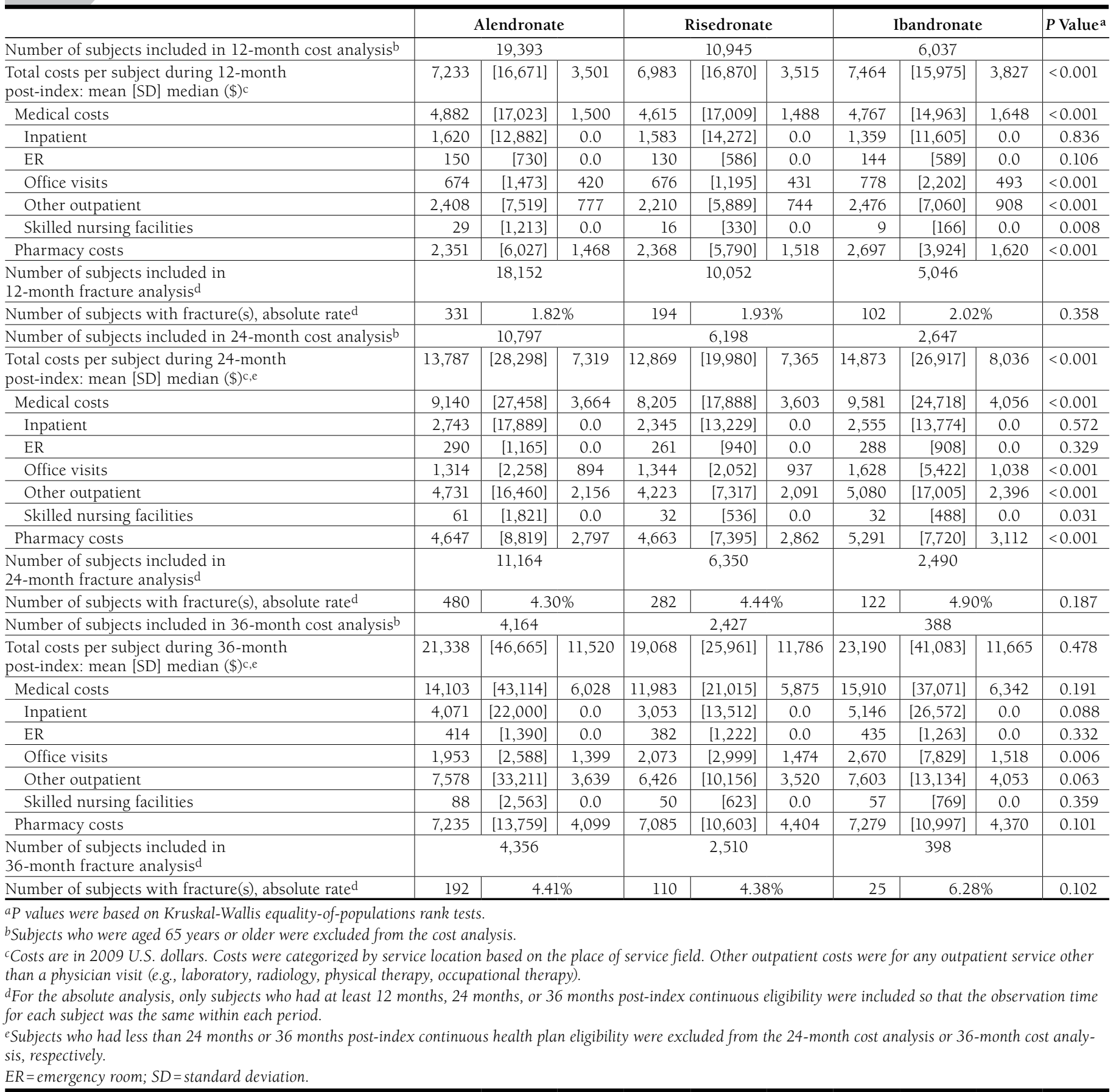

oral bisphosphonates. The more than 45,000 subjects in the present study consisted of a geographically diverse population enrolled in a variety of health plans and can be considered representative of members eligible to receive bisphosphonate therapy in a managed care setting.
Adherence to osteoporosis medications in the present study, as measured by MPR, ranged from 0.44 to 0.58 . There was no significant difference in adherence among the individual bisphosphonates in the first 12 months. By the end of 24 months, the rate of adherence had dropped for all 3 


\section{Analysis of the Comparative Effectiveness of 3 Oral Bisphosphonates in a Large Managed Care Organization: Adherence, Fracture Rates, and All-Cause Cost}

\section{TABLE 5 Number of Persons with Fracture(s) per Month}

\begin{tabular}{l|c|c|c|c|c|c|c}
\hline & $\mathbf{n}$ & $\begin{array}{c}\text { Mean Follow-Up } \\
\text { (Months) }\end{array}$ & $\begin{array}{c}\text { Total Person-Months } \\
\text { of Follow-Up }\end{array}$ & $\begin{array}{c}\text { Number of } \\
\text { Fractures }\end{array}$ & $\begin{array}{c}\text { Number of Fractures } \\
\text { Per Patient-Month }\end{array}$ & $\begin{array}{c}P \text { Value Versus } \\
\text { Alendronate }^{\mathbf{a}}\end{array}$ & $\begin{array}{c}\boldsymbol{P} \text { Value Versus } \\
\text { Ibandronate }^{\mathbf{a}}\end{array}$ \\
\hline Alendronate & 21,802 & 24.76 & 539,818 & 1,058 & 0.0020 & - \\
\hline Risedronate & 12,062 & 25.11 & 302,877 & 648 & 0.0021 & 0.080 \\
\hline Ibandronate & 6,316 & 21.27 & 134,341 & 296 & 0.0022 & 0.083 \\
\hline
\end{tabular}

\section{TABLE 6 Generalized Linear Regression Model of All-Cause Health Care Costs During 12, 24, and 36 Months Post-Index, Among Incident Osteoporosis Subjects}

\begin{tabular}{|c|c|c|c|c|c|c|}
\hline Adjusted Results & $\operatorname{Exp}(b)^{a}(95 \% C I)$ & $P$ Value & $\operatorname{Exp}(b)^{a}(95 \% C I)$ & $P$ Value & $\operatorname{Exp}(b)^{a}(95 \%$ CI $)$ & $P$ Value \\
\hline & \multicolumn{2}{|c|}{ 12-Month Results } & \multicolumn{2}{|c|}{ 24-Month Results } & \multicolumn{2}{|c|}{ 36-Month Results } \\
\hline \multicolumn{7}{|l|}{ Osteoporosis medication at index } \\
\hline Alendronate ${ }^{\mathrm{b}}$ & Reference & & Reference & & Reference & \\
\hline Risedronate $^{b}$ & $0.992(0.955-1.032)$ & 0.701 & $0.991(0.936-1.050)$ & 0.763 & $0.993(0.880-1.121)$ & 0.911 \\
\hline Ibandronate ${ }^{b}$ & $1.023(0.975-1.073)$ & 0.361 & $0.969(0.911-1.030)$ & 0.315 & $0.953(0.842-1.079)$ & 0.450 \\
\hline \multicolumn{7}{|l|}{ Baseline characteristics } \\
\hline \multicolumn{7}{|l|}{ Age (years) } \\
\hline $45-54$ & Reference & & Reference & & Reference & \\
\hline $55-64$ & $1.047(1.012-1.084)$ & 0.009 & $1.039(1.000-1.080)$ & 0.050 & $1.019(0.964-1.077)$ & 0.506 \\
\hline \multicolumn{7}{|l|}{ Gender } \\
\hline Male & Reference & & Reference & & Reference & \\
\hline Female & $0.698(0.648-0.753)$ & $<0.001$ & $0.746(0.686-0.810)$ & $<0.001$ & $0.626(0.555-0.705)$ & $<0.001$ \\
\hline \multicolumn{7}{|l|}{ Region } \\
\hline East & Reference & & Reference & & Reference & \\
\hline South & $0.942(0.892-0.994)$ & 0.030 & $0.907(0.854-0.963)$ & 0.002 & $0.944(0.865-1.030)$ & 0.192 \\
\hline Central & $0.983(0.926-1.044)$ & 0.573 & $0.924(0.864-0.988)$ & 0.020 & $0.886(0.806-0.974)$ & 0.012 \\
\hline West & $0.906(0.860-0.954)$ & $<0.001$ & $0.895(0.845-0.948)$ & $<0.001$ & $0.863(0.795-0.936)$ & $<0.001$ \\
\hline \multicolumn{7}{|l|}{ Health plan type } \\
\hline $\mathrm{HMO}$ & Reference & & Reference & & Reference & \\
\hline POS & $1.123(1.044-1.207)$ & 0.002 & $1.122(1.036-1.216)$ & 0.005 & $1.208(1.079-1.353)$ & 0.001 \\
\hline $\mathrm{PPO}$ & $1.098(1.054-1.144)$ & $<0.001$ & $1.128(1.077-1.181)$ & $<0.001$ & $1.120(1.046-1.200)$ & 0.001 \\
\hline FFS & $1.193(0.980-1.452)$ & 0.078 & $1.225(0.990-1.514)$ & 0.061 & $1.073(0.807-1.427)$ & 0.628 \\
\hline Other & $1.171(1.062-1.290)$ & 0.002 & $1.153(1.037-1.283)$ & 0.009 & $1.141(0.973-1.338)$ & 0.104 \\
\hline \multicolumn{7}{|l|}{ DCI score, 12 months pre-index } \\
\hline 0 & Reference & & Reference & & Reference & \\
\hline 1 & $1.426(1.360-1.495)$ & $<0.001$ & $1.410(1.338-1.486)$ & $<0.001$ & $1.469(1.361-1.586)$ & $<0.001$ \\
\hline 2 & $1.780(1.609-1.970)$ & $<0.001$ & $1.683(1.501-1.886)$ & $<0.001$ & $1.640(1.397-1.925)$ & $<0.001$ \\
\hline 3 or more & $1.804(1.593-2.043)$ & $<0.001$ & $1.602(1.385-1.853)$ & $<0.001$ & $1.491(1.188-1.873)$ & 0.001 \\
\hline Pre-index osteoporosis diagnosis & $1.105(1.067-1.143)$ & $<0.001$ & $1.096(1.054-1.138)$ & $<0.001$ & $1.098(1.039-1.161)$ & 0.001 \\
\hline Pre-index arthritis (OA and RA) diagnosis & $1.301(1.248-1.357)$ & $<0.001$ & $1.294(1.234-1.357)$ & $<0.001$ & $1.314(1.226-1.408)$ & $<0.001$ \\
\hline Pre-index musculoskeletal pain diagnosis & $1.267(1.214-1.321)$ & $<0.001$ & $1.229(1.173-1.288)$ & $<0.001$ & $1.191(1.113-1.275)$ & $<0.001$ \\
\hline Pre-index total cost (2008 U.S. $\$ 1,000$ s) & $1.032(1.030-1.035)$ & $<0.001$ & $1.035(1.032-1.038)$ & $<0.001$ & $1.030(1.026-1.033)$ & $<0.001$ \\
\hline \multicolumn{7}{|c|}{$\begin{array}{l}{ }^{a} \text { Coefficients were exponentiated because a log link was used in the GLM regression; retransformation into exp }(b) \text { facilitated interpretation. For example, an exp }(b)=0.99 \\
\text { means that the costs for a group are } 99 \% \text { of the costs for the reference group. } \\
\text { bNumber of subjects at each time period is the same as those included in the respective cost analyses in Table } 4 . \\
C I=\text { confidence interval; } D C I=\text { Deyo-Charlson Comorbidity Index; } \exp (b)=\text { exponentiated coefficient; } F F S=f e e \text { for service; GLM=generalized linear model; HMO=health } \\
\text { maintenance organization; OA= osteoarthritis; POS = point of service; } P P O=\text { preferred provider organization; RA = rheumatoid arthritis. }\end{array}$} \\
\hline
\end{tabular}

medications. Although MPR was statistically significantly less with ibandronate compared with either alendronate or risedronate, the clinical significance of this small difference is unknown. This finding was surprising, given that ibandronate is usually taken once per month. Again, at 36 months, the rate of adherence for all 3 medications had dropped further. In conclusion, the rates of adherence to the bisphosphonates in this study can be considered to be similar. 


\section{Analysis of the Comparative Effectiveness of 3 Oral Bisphosphonates in a Large Managed Care Organization: Adherence, Fracture Rates, and All-Cause Cost}

Alendronate users had a statistically significant $12 \%$ lower risk of experiencing at least 1 fracture than ibandronate users, after controlling for other subject characteristics. There was no significant difference between risedronate and ibandronate. The unadjusted fracture rate differences were small and nonsignificant; however, the adjusted fracture risk may be a more appropriate measure for this type of analysis. It accounts for differences in subject follow-up period. For the absolute analysis of percentage of subjects with fracture, only subjects who had at least 12-month, 24-month, or 36-month post-index continuous eligibility were included so that the observation time for each subject was the same within each period.

When comparing costs among the 3 oral bisphosphonates, subjects on ibandronate incurred higher costs than those using the other 2 drugs, both in terms of total health care costs and costs by service location. A $\$ 231$ difference in mean total health care costs between alendronate users and ibandronate users and a $\$ 481$ difference in mean total health care costs between risedronate users and ibandronate users were observed during the first 12 months post-index. Larger differences in total health care costs were seen in longer follow-up periods (\$1,086 and \$2,004 over 24 months; $\$ 1,851$ and $\$ 4,122$ over 36 months, respectively). However, the adjusted differences among the 3 medication groups became insignificant, which suggests that other factors may explain the observed cost difference. According to the regression model, older age, male gender, East region, higher DCI score, pre-index diagnosis of arthritis or musculoskeletal pain, and higher pre-index total costs were associated with higher total costs. The results of sensitivity analyses were consistent with these results.

Others have evaluated the costs of treating osteoporosis and osteoporosis-related fracture. Not surprisingly, a database review by Desai et al. (2003) concluded that for women aged 45-65 years enrolled in an HMO the costs of treating an osteoporosis-related fracture ( $\$ 939$ per patient per year [PPPY]) were significantly higher than the costs of treating osteoporosis only (\$645 PPPY). Costs were also significantly higher for women not receiving drug therapy for osteoporosis (\$724 PPPY) than for those who were ( $\$ 679$ PPPY). These results suggest that preventive treatment costs may offset the costs of treating established disease. ${ }^{15}$

Previous observational studies have looked at fracture risk. Harris et al. (2009) found that rates of hip, nonvertebral, or any clinical fracture rate did not significantly differ between monthly ibandronate and weekly alendronate or risedronate in adherent (no discontinuation of bisphosphonate within 90 days of index date) women aged 45 years or older receiving up to 1 year of treatment; however, rates of vertebral facture were lower with ibandronate. ${ }^{11}$ Weekly risedronate was associated with lower rates of hip and nonvertebral fractures than weekly alendronate in women aged 65 years or older in the first year following treatment in an analysis by Silverman et al. (2007). ${ }^{10}$
These results should be interpreted with caution because continuous enrollment in the health plan was not required in the analysis by Silverman et al., leading to significant censoring of the data. In another database analysis by Watts et al. (2004), risedronate was found to be significantly more effective than alendronate within 12 months of treatment (absolute risk 0.9\% vs. $2.4 \%$, adjusted relative risk reduction $59 \%, P=0.04$ ) for nonvertebral fractures in men and women aged 45 years or older who had at least 2 prescriptions filled for the index drug within the first 45 days. ${ }^{12}$ Low adherence has been associated with both higher risk of fracture and higher costs. ${ }^{13,14,16}$ For instance, in the analysis by Halpern et al. (2011), commercially insured women with low adherence (MPR < 80\%) had 37\% higher fracture risk and $12 \%$ higher all-cause medical costs through 18 months of follow-up compared with women with higher adherence (MPR > 80\%). ${ }^{16}$

The differences in methodology of these and other studies make it difficult to compare their results with ours. Also, it is questionable whether one can draw conclusions regarding relative effectiveness from studies of 12-18 months duration in a chronic disease such as osteoporosis.

Health care reform, known as the Patient Protection \& Affordable Care Act (PPACA), has mandated changes in the delivery system, requiring a focus on both cost and quality of care. Funding has also been put in place for expansion of CER. Integrated care, with the coordination of medical and pharmacy benefits for the prevention and management of chronic illness, can play an essential role in reform. Determining realworld outcomes through the use of integrated database reviews can provide important insights into the most effective use of drug therapies.

The results from this large database analysis were a meaningful component of the formulary discussions surrounding the differences between the bisphosphonates and their expected impact on patient care. They provided additional insights into real-world outcomes, including quantification of total costs and their shift between pharmacy and medical.

\section{Limitations}

First, retrospective observational studies lack randomization of the intervention. Bias can result from the subject selection process, how subjects are allocated to treatment, variability in how subjects are treated, and other confounding factors. Statistical adjustment can reduce the impact of observable factors but cannot eliminate the impact of unobserved factors (e.g., family history, smoking history, fall risk) that are not available in an administrative claims database. Second, members were included in the analysis even if they had only 1 claim for an osteoporosis medication during the intake period. This may not seem to be a robust criterion, but it does reflect behavior in a real-world population.

Third, adherence, compliance, and even MPR have not been 


\section{Analysis of the Comparative Effectiveness of 3 Oral Bisphosphonates in a Large Managed Care Organization: Adherence, Fracture Rates, and All-Cause Cost}

consistently defined in the literature, and various methods have been proposed to calculate these measures. ${ }^{17,20-22,25}$ Each has its own strengths and weaknesses, where confounders within each measure may significantly affect results. Although the MPR is a common measure of adherence, it does not address the consistency of refills or whether people are actually taking the medication as directed.

Fourth, differences in member cost-share and ability to afford the medications, as well as subjects receiving samples from their physicians, could have affected the adherence rates and contributed to differences among the agents. During the study period, there were differences in the copayment structure for the oral bisphosphonate agents. Throughout most of the study period, brand alendronate and risedronate were considered preferred agents, while ibandronate was on a highercost tier. Although all 3 agents were formulary, the typical copayment for the preferred agents was approximately $\$ 10$, while that for ibandronate was approximately $\$ 35$. This could account for fewer subjects utilizing ibandronate. Fifth, generic alendronate became available during the course of the study in February 2008. Our study did not stratify by brand versus generic utilization. Utilization of generic alendronate would have resulted in lower pharmacy cost figures and consequently would have reduced the total cost figures for alendronate compared with the other agents. Since the observation time period (12 months post-index) for more than $80 \%$ of the subjects did not go beyond 2007, the impact of generic availability of alendronate should be minor.

Sixth, subjects were not stratified by dosing frequency (i.e., daily, weekly, or monthly). However, the vast majority of subjects were using weekly alendronate ( $>98 \%)$ and risedronate $(>95 \%)$ and monthly ibandronate (>99\%). There was no control for differences in these variables in the analyses. The relationship of dosing frequency on adherence to osteoporosis medications is complex, and it has been suggested that it may not be the most important consideration in treatment decisions. ${ }^{26}$ In addition, when examining the association between the different oral bisphosphonates and fracture rates, the influence of medication adherence was not assessed, since the relationship has been well established by previous studies.

Seventh, potential measurement misclassification and incorrect coding of the fracture events are possible limitations of a claims database analysis in which patient charts are not available to verify the diagnosis. A stringent definition was used in identifying incident fractures with claims data in this study to minimize such measurement error. ${ }^{23}$ Additionally, only those fractures for which medical attention was sought were recorded, but unrecorded fracture events would be expected to be distributed evenly among the 3 medication groups. The analysis also did not account for osteoporosis severity or type of fracture (e.g., hip vs. vertebral).

Finally, exclusion of the cost data in the group of individuals aged 65 years or older limits generalizability to this age group. The proportions of subjects aged 65 years or older were $22 \%$, $21 \%$, and $16 \%$ in the alendronate, risedronate, and ibandronate cohorts, respectively. Many of these older subjects were Medicare beneficiaries, and it is difficult to characterize their total cost picture.

\section{Conclusions}

This retrospective analysis of an administrative claims database in a large managed care population showed similar rates of adherence and total adjusted all-cause health care costs for alendronate, risedronate, and ibandronate. Absolute unadjusted rates of fracture were small and did not significantly differ among agents, but after adjusting for subject characteristics, alendronate was associated with a $12 \%$ lower risk of experiencing any incident fracture compared with ibandronate. Observational evaluations of this type will become increasingly more important and useful as decision makers strive to differentiate among competing medications.

\section{Authors}

KAREN E. MARTIN, PharmD, MBA, CGP, is Senior Drug Information Specialist; H. ELOISE CAMPBELL, BS, PharmD, is Senior Drug Information Specialist; JACOB ABARCA, PharmD, MS, is Outcomes Research Scientist; and T. JEFFREY WHITE, PharmD, MS, is Director, Drug Evaluation and Clinical Analytics, WellPoint, Inc., Indianapolis, Indiana. JINGBO YU, MHA, PhD, is Manager, Global Health Outcomes, Merck \& Company, Inc., Whitehouse Station, New Jersey.

AUTHOR CORRESPONDENCE: Karen E. Martin, PharmD, MBA, CGP, WellPoint, Inc., 1757 Phillips Rd., Lebanon, OH 45036.

Tel.: 513.933.0436; E-mail: karen.martin-OH@wellpoint.com.

\section{DISCLOSURES}

There was no outside funding for this study. Concept and design were performed by White with the assistance of Abarca and Yu. Data were collected by $\mathrm{Yu}$ with the assistance of Abarca and White and analyzed primarily by Martin and $\mathrm{Yu}$. The manuscript was written by Martin with the assistance of Campbell and Yu and revised primarily by Martin with the assistance of Abarca.

\section{ACKNOWLEDGEMENTS}

The authors would like to acknowledge the work of John Barron, PharmD; An-Chen Fu, MS; and Ralph Turner, PhD, HealthCore, Inc., who performed data analysis. 


\section{Analysis of the Comparative Effectiveness of 3 Oral Bisphosphonates in a Large Managed Care Organization: Adherence, Fracture Rates, and All-Cause Cost}

\section{REFERENCES}

1. National Osteoporosis Foundation. Fast facts. Available at: www.nof.org/ node/40. Accessed September 6, 2011.

2. National Osteoporosis Foundation. NOF clinician's guide to prevention and treatment of osteoporosis. 2010. Available at: http://www.nof.org/professionals/clinical-guidelines. Accessed September 6, 2011.

3. Management of osteoporosis in postmenopausal women. 2010 position statement of The North American Menopause Society. Menopause. $2010 \cdot 17(1): 25-54$

4. Watts NB, Bilezikian JP, Camacho PM, et al. American Association of Clinical Endocrinologists Medical Guidelines for Clinical Practice for the diagnosis and treatment of postmenopausal osteoporosis. Endocr Pract. 2010;16(Suppl 3):S1-S37

5. Papapoulos SE, Quandt SA, Liberman UA, Hochberg MC, Thompson DE. Meta-analysis of the efficacy of alendronate for the prevention of hip fractures in postmenopausal women. Osteoporos Int. 2005;16(5):468-74.

6. Black DM, Cummings SR, Karpf DB, et al. Randomised trial of effect of alendronate on risk of fracture in women with existing vertebral fractures. Fracture Intervention Trial Research Group. Lancet. 1996;348(9041):153541

7. Cummings SR, Black DM, Thompson DE, et al. Effect of alendronate on risk of fracture in women with low bone density but without vertebral fractures: results from the Fracture Intervention Trial. JAMA. 1998;280(24):2077-82. Available at: http://jama.ama-assn.org/content/280/24/2077.long. Accessed September 6, 2011

8. Harris ST, Watts NB, Genant HK, et al. Effects of risedronate treatment on vertebral and nonvertebral fractures in women with postmenopausal osteoporosis: a randomized controlled trial. Vertebral Efficacy With Risedronate Therapy (VERT) Study Group. JAMA. 1999;282(14):1344-52. Available at: http://jama.ama-assn.org/content/282/14/1344.long. Accessed September 6, 2011

9. Chesnut III CH, Skag A, Christiansen C, et al. Effects of oral ibandronate administered daily or intermittently on fracture risk in postmenopausal osteoporosis. J Bone Miner Res. 2004;19(8):1241-49.

10. Silverman SL, Watts NB, Delmas PD, Lange JL, Lindsay R. Effectiveness of bisphosphonates on nonvertebral and hip fractures in the first year of therapy: the risedronate and alendronate (REAL) cohort study. Osteoporos Int. 2007;18(1):25-34. Available at: http://www.ncbi.nlm.nih.gov/pmc/ articles/PMC1705543/pdf/198_2006_Article_274.pdf. Accessed September 6,2011

11. Harris ST, Reginster JY, Harley C, et al. Risk of fracture in women treated with monthly oral ibandronate or weekly bisphosphonates: The eValuation of IBandronate Efficacy (VIBE) database fracture study. Bone. 2009:44(5):758-65.

12. Watts NB, Worley K, Solis A, Doyle J, Sheer R. Comparison of risedronate to alendronate and calcitonin for early reduction of nonvertebral fracture risk: results from a managed care administrative claims database. J Manag Care Pharm. 2004;10(2):142-51. Available at: http://www.amcp.org/ data/jmcp/Formulary\%20Management-142-151.pdf.
13. Siris ES, Harris ST, Rosen CJ, et al. Adherence to bisphosphonate therapy and fracture rates in osteoporotic women: relationship to vertebral and nonvertebral fractures from 2 US claims databases. Mayo Clin Proc. 2006;81(8):1013-22. Available at: http://www.mayoclinicproceedings.com/ content/81/8/1013.full.pdf+html. Accessed September 6, 2011.

14. Huybrechts KF, Ishak KJ, Caro JJ. Assessment of compliance with osteoporosis treatment and its consequences in a managed care population. Bone. 2006;38(6):922-28

15. Desai SS, Duncan BS, Sloan AS. The cost of treating osteoporosis in a managed health care organization. J Manag Care Pharm. 2003;9(2):142-49. Available at: http://www.amcp.org/data/jmcp/Research-142-149.pdf.

16. Halpern R, Becker L, Iqbal SU, Kazis LE, Macarios D, Badamgarav E. The association of adherence to osteoporosis therapies with fracture, allcause medical costs, and all-cause hospitalizations: a retrospective claims analysis of female health plan enrollees with osteoporosis. J Manag Care Pharm. 2011;17(1):25-39. Available at: http://www.amcp.org/WorkArea/ DownloadAsset.aspx?id=8963

17. Sun P, Zhao Y, Ray S. How to improve the quality of medication adherence estimates derived from healthcare claims or electronic medical records databases. Presented at: The 14th Annual International Meeting of the International Society of Pharmacoeconomics and Outcomes Research (ISPOR); May 16-20, 2009; Orlando, FL.

18. Zhao Y, Sun P, Watson P. Medication adherence and healthcare costs among patients with diabetic peripheral neuropathic pain initiating duloxetine versus pregabalin. Curr Med Res Opin. 2011;27(4):785-92.

19. Zhao Y, Sun P, Watson P, Mitchell B, Swindle R. Comparison of medication adherence and healthcare costs between duloxetine and pregabalin initiators among patients with fibromyalgia. Pain Pract. 2011;11(3):204-16.

20. Cramer JA, Roy A, Burrell A, et al. Medication compliance and persistence: terminology and definitions. Value Health. 2008;1(1)1:44-47.

21. LaFleur J, Oderda GM. Methods to measure patient compliance with medication regimens. J Pain Palliat Care Pharmacother. 2004;18(3):81-87.

22. Peterson AM, Nau DP, Cramer JA, et al. A checklist for medication compliance and persistence studies using retrospective databases. Value Health. 2007;10(1):3-12

23. Warriner AH, Patkar NM, Curtis JR, et al. Which fractures are most attributable to osteoporosis? J Clin Epidemiol. 2011;64(1):46-53.

24. Deyo RA, Cherkin DC, Ciol MA. Adapting a clinical comorbidity index for use with ICD-9-CM administrative databases. J Clin Epidemiol. 1992;45(6):613-19.

25. Hess LM, Raebel MA, Conner DA, Malone DC. Measurement of adherence in pharmacy administrative databases: a proposal for standard definitions and preferred measures. Ann Pharmacother. 2006;40(7-8):1280-88.

26. Lee S, Glendenning P, Inderjeeth CA. Efficacy, side effects and route of administration are more important than frequency of dosing of anti-osteoporosis treatments in determining patient adherence: a critical review of published articles from 1970 to 2009. Osteoporos Int. 2011;22(3):741-53. 


\section{Analysis of the Comparative Effectiveness of 3 Oral Bisphosphonates in a Large Managed Care Organization: Adherence, Fracture Rates, and All-Cause Cost}

\section{APPENDIX Codes for Exclusion Criteria, Type of Fracture, Procedure, Comorbidities, and Medications}

Description

Exclusion criteria

Paget's disease

Neoplasm

Type of fracture

Dorsal and lumbar vertebral fractures

Hip, pelvis, femur, lower forearm, radius/ulna and humerus

Other fractures

Procedure codes for fracture
Type of Code

\begin{tabular}{|l|l} 
ICD-9-CM diagnosis & 731.xx \\
\hline
\end{tabular}

\begin{tabular}{l|l} 
ICD-9-CM diagnosis & $140 . \mathrm{xx}-208 . \mathrm{xx}$
\end{tabular}

ICD-9-CM diagnosis ICD-9-CM diagnosis ICD-9-CM diagnosis

ICD-9-CM diagnosis CPT
Other vertebral fractures

$733.13,805.2 \mathrm{x}, 805.4 \mathrm{x}$

805.0x, 805.1x, 805.3x, 805.6x, 805.7x, 805.8x, 805.9x, 806.xx

$733.11,733.12,733.14,733.15,808 . x, 812 . x x, 813 . x, 813.1 x, 813.2 x, 813.3 x, 813.4 x, 813.5 x$,

813.8x, 813.9x, 814.xx, 820.xx, 821.xx

733.16, 807.xx, 809.x, 810.xx, 811.xx, 823.xx

22520-22522, 76012, 76013, 22305, 22310, 22315, 22318, 22319, 22325, 22326, 22327, 22328 , 72010-72159, 72240-72295 (radiology), 27230-27248, 27193-27194, 27215-27218, 27220,

$27222,27226-27228,27500-27514,24650,24655,24665,24666,24670,24675,24685,25500$,

$25505,25515,25520,25525,25526,25530,25535,25545,25560,25565,25574,25575,25600$,

$25605,25611,25620,25650,25651,25652,25622,25624,25628,25630,25635,25645$

$25680,25685,23600,23605,23615,23616,23620,23625,23630,23665,24500,24505$,

$24515,24516,24530,24535,24538,24545,24546,24560,24565,24566,24575,24576,24577$, $24579,24582,76012,76013$

Pre-index diagnoses codes

Osteoporosis

Medical claim for BMD test

Osteopenia

Arthritis

Musculoskeletal pain

Menopause and menopausal symptoms

Respiratory diseases

Hypothyroidism

Dementia, depression, anxiety, sleep disorders

Diabetes

Upper gastrointestinal disorders

Cardiovascular disease

Cerebrovascular disease

Indication of decreased mobility

Kidney disease

Nutritional disorder

Hyperthyroidism

Obesity

Hyperparathyroidism

Alzheimer's disease

Parkinson's disease

Alcohol use disorder

Bulimia or anorexia

Medications

Alendronate

Risedronate

Ibandronate

Cardiovascular medication

Analgesics

Central nervous system agents

Gastrointestinal medication

Thyroid hormones

Estrogen

Corticosteroids

Respiratory agents

Antidiabetic medication

Antineoplastic hormonal agents

Smoking cessation medication

ICD-9-CM diagnosis

CPT

ICD-9-CM diagnosis

ICD-9-CM diagnosis

ICD-9-CM diagnosis

ICD-9-CM diagnosis

ICD-9-CM diagnosis

ICD-9-CM diagnosis

ICD-9-CM diagnosis

ICD-9-CM diagnosis

ICD-9-CM diagnosis

ICD-9-CM diagnosis

ICD-9-CM diagnosis HCPCS

ICD-9-CM diagnosis ICD-9-CM diagnosis ICD-9-CM diagnosis ICD-9-CM diagnosis ICD-9-CM diagnosis ICD-9-CM diagnosis ICD-9-CM diagnosis ICD-9-CM diagnosis ICD-9-CM diagnosis

\begin{tabular}{|l|l|}
\hline GPI-8 & 30012010 \\
\hline GPI-8 & 30042065 \\
\hline GPI-8 & 30042048 \\
\hline GPI-2 & $31-40$ \\
\hline GPI-2 & $64-66$ \\
\hline GPI-2 & $57-60,62,72-73$ \\
\hline GPI-2 & $46-52$ \\
\hline GPI-4 & 2810 \\
\hline GPI & $24000015-24000017,24000030,24991002,24993002,240000350003,24000060$, \\
\hline GPI-2 & 240000350088 \\
\hline GPI-2 & 22 \\
\hline GPI-4 & $44-45$ \\
\hline GPI-4 & $2710,2715,2717,2720,2723,2725,2728,2750,2755,2760,2799$ \\
\hline GPI-6 & 2140 \\
\hline
\end{tabular}

BMD=bone mineral density; CPT=Current Procedural Terminology; GPI=generic product identifier (Medi-Span); HCPCS=Healthcare Common Procedure Coding System; ICD-9CM=International Classification of Diseases, Ninth Revision, Clinical Modification
$733.0 \mathrm{x}$

$76070,76071,76075,76076,76078,76977,77078-77081,77083$

733.9

714.0x, 715.xx, 716.xx, 720.0x, 721.0x, 721.2x, 721.3x, 721.9x, 729.0x, 729.1x, 729.2x, 729.5x, $729.9 \mathrm{x}$

$721-723,724 . x, 739.1,739.2,739.3,739.4$

256.31, 627.xx

472.xx, 473.xx, 476.xx, 477.xx, 478.xx, 490.xx, 491.xx, 492.xx, 493.xx, 494.xx, 496.xx, 511.

$\mathrm{xx}, 515 . \mathrm{xx}, 516 . \mathrm{xx}, 518.1 \mathrm{x}, 518.2 \mathrm{x}, 518.3 \mathrm{x}, 518.83,519.2 \mathrm{x}, 519.9 \mathrm{x}$

243.xx, $244, \mathrm{xx}$

290.xx, 294.1x, 294.8x, 296.2x, 296.3x, 298.0x, 300.0x, 307.4x, 309.1x, 311.xx, 331.1x, 780.5x

$250 . x x$

530.1x, 530.2x, 530.81, 531.xx, 533.xx, 534.xx, 535.xx, 578.x, 787.2x

410.xx-414.xx

433.xx-438.xx

E1050-E1298, E1031-E1035, E0100-E0105, E0110-E0118, E0130-E0135, L5000-L5999, K0001-K0732

250.4x, 403.xx, 404.xx, 405.01, 405.11, 405.91, 580.xx-589.xx, 753.0x, 753.1x, 791.0x

263.xx-269.xx, 579.0x, 579.2x, 579.9x

$242.0 \mathrm{x}-242.3 \mathrm{x}, 242.9 \mathrm{x}$

$278.0 \mathrm{x}, 649.1 \mathrm{x}$

$252.0 x, 259.3 \mathrm{x}$

$331.0 \mathrm{x}$

$332 . \mathrm{xx}$

291.xx, 303.xx, 571.0x-571.3x

$307.1 \mathrm{x}, 307.51,783.0 \mathrm{x}$

\section{0}

30042048

$57-60,62,72-73$

$24000015-24000017,24000030,24991002,24993002,240000350003,24000060$ 240000350088

$44-45$

2140 